\title{
The pharmacotherapy of low back pain
}

\author{
Prof Oppel B W Greeff, MBChB, FCFP(SA), MPharmMed, FFPM(RCP), MD
}

Medical Director: Acquipharma

\section{Introduction}

About $60-80 \%$ of patients visiting their family practitioner have at some stage in their lives suffered from low back pain. The annual incidence in adults aged 35 -55years in developed countries is up to $45 \%$. The differential diagnosis is broad and includes muscular strain, primary spine disease like disc herniation or degenerative arthritis, systemic diseases like metastatic cancer and regional diseases like aortic aneurisms. In the majority of cases, a specific diagnosis can not be made.

Most patients will improve in $1-4$ weeks and will only need treatment for the acute symptoms after the initial history and physical examination. If, however, the pain recurs or gets worse, the patient must be thoroughly examined and specific diagnosis can become a challenge.

\section{Causes of Lower Back Pain}

There are multiple causes of lower back pain and the physician will always attempt toget to the correct cause in order to assure the most effective treatment is recommended.Modern imaging techniques have contributed significantly to getting to the correct diagnosis. The following conditions may cause lower back pain: Cancer, Cauda Equina Syndrome, Herniated intervertebral disc, severe or progressive neurological deficits, spinal stenosis, vertebral compression fracture or vertebral infection

When examining and communicating with the patient, the practitioner must take special care if the patient complains of severe back pain that:

1. Followed a fall, blow to the back or other injury

2. Is constant or severe

3. Worsens during rest or at night

4. Spreads down one or both legs

5. Causes weakness, numbness or tingling in one or both legs

6. Is associated with new bowel or bladder problems

7. Is accompanied by fever

8. Is associated with pain or throbbing in the abdomen

9. Is accompanied by unexplained weight loss
This will ensure that serious causes of lower back pain is not overseen.

\section{Management of Lower Back Pain}

The state of Oregon in the USA recently published guidelines for the evaluation and management of low back pain. The following table gives a good summary of the current medical practice.

From this table it is apparent that self-care, nonpharmacologic therapy and interdisciplinary therapy are important in the in the total intervention programme. We will focus on the pharmacologic therapy of Lower back pain.

\section{Pharmacotherapy of Lower Back Pain}

The most commonly used medicines in the treatment of lower back pain are the analgesics. Therapy will be discussed as follow:

1. Non-Opioid Analgesics

2. Opioids

3. Skeletal muscle relaxants

4. Other Central Nervous System drugs

\section{The Non-Opioid Analgesics:}

- Paracetamol (Acetaminophen) is the most commonly used and prescribed analgesic for lower back pain. It has equivalent analgesic efficacy to aspirin with no useful anti-inflammatory action. Paracetamol has a central anti-nociceptive effect through selective inhibition of prostaglandin $\mathrm{H} 2$ synthetase. It serves as a reducing co-substrate for the peroxidase-active site of the enzyme (= a -POX inhibitor) It is conjugated in the liver as the inactive glucoronate and sulphate. Adverse effects are rare in therapeutic usage - occasional rash and allergy. Dosage orally should not exceed 4G/day and intra-venously a loading dose of $2 \mathrm{~g}$, followed by $1 \mathrm{G}$ 4-hourly to a maximum of 4g/Day)PERFALGAN)

- Aspirin - is the oldest and most well- known and widely used analgesic. It has analgesic, anti-pyretic and antiinflammatory actions and causes respiratory stimulation. It can cause respiratory alkalosis, renal loss of electrolytes 
Table I: Interventions

\begin{tabular}{|c|c|c|c|}
\hline Intervention category & Intervention & Acute $<4$ weeks & Subacute and chronic $>4$ weeks \\
\hline \multirow[t]{2}{*}{ Self-care } & Advice to remain active & $\bullet$ & $\bullet$ \\
\hline & Application of superficial heat & $\bullet$ & \\
\hline \multirow[t]{7}{*}{ Nonpharmacologic therapy } & Spinal manipulation & $\bullet$ & $\bullet$ \\
\hline & Exercise therapy & & $\bullet$ \\
\hline & Massage & & $\bullet$ \\
\hline & Acupuncture & & $\bullet$ \\
\hline & Yoga & & $\bullet$ \\
\hline & Cognitive-behavioural therapy & & $\bullet$ \\
\hline & Progressive relaxation & & $\bullet$ \\
\hline \multirow{6}{*}{$\begin{array}{l}\text { Pharamacologic therapy } \\
\text { (Carefully consider risks/harms) }\end{array}$} & Acetaminophen & $\bullet$ & • \\
\hline & NSAIDs & - $\boldsymbol{\Delta}$ & - $\Delta$ \\
\hline & $\begin{array}{l}\text { Skeletal muscle relaxants e.g. } \\
\text { orphenadrine, methocarbamol and } \\
\text { cyclobenzaprine }\end{array}$ & $\bullet$ & \\
\hline & Antidepressants (TCA) & & $\bullet$ \\
\hline & Benzodiazepines & - $\Delta$ & - $\boldsymbol{\Delta}$ \\
\hline & Tramadol, opioids & $\bullet \boldsymbol{\Delta}$ & - $\boldsymbol{\Delta}$ \\
\hline Interdisciplinary therapy & Intensive interdisciplinary rehabilitation & & $\bullet$ \\
\hline
\end{tabular}

- Interventions supported by grade "B" evidence (at least fair-quality evidence of moderate benefit, or small benefit but no significant harms, costs, or burdens). No intervention was supported by grade " $\mathrm{A}$ " evidence (good-quality evidence of substantial benefit).

$\Delta$ Carries greater risk of harms than other agents in table.

with dehydration plus a disturbance of glucose metabolism. Aspirin has a uricosuric effect in dosages between 5-8g/day. It reduces platelet adhesion and causes hypothrombobinaemia in dosages $>5 G /$ day. The normal dosage of aspirin is $325-$ 650mg every 4-6hours for pain and fever.

- The Non-Steroidal Anti-Inflammatory Drugs(NSAID's)

- The NSAID's inhibit cyclo-oxygenase(prostaglandin synthase) that is responsible for conversion of arachidonic acid to cyclic endoperoxidases. There are 2 iso-forms - COX-1 and 2, which led to the sub-group of COX-2 selective inhibitors. Their main actions are analgesic, anti-inflammatory, anti-pyretic and antiplatelet(prevent thromboxane production) The side-effects include gastric and intestinal mucosal damage, disturbances of fluid and elecftrolyte balance and they can cause analgesic nephropathy.Dosages vary in the different sub-classes.

\section{The Opioids}

There are 4 types of G-protein-coupled opioid receptors, each having a different pharmacological effect - mu, kappa, delta and the ORL-1 receptors. The affinity for these receptors parallels their analgesic potency. Morphine is the prototype opioid and has the following effects: Analgesia, euphoria, respiratory depression, depression of the cough reflex, nausea and vomiting and pupillary constriction. It also has significant effects on the GIT - increased tone, decreased motility, constriction of the biliary sphincter and delayed absorption of other drugs. Morphine is used for severe or overwhelming pain. Oral dosage is $30 \mathrm{mg}$ every 3-4hours and 10mg Q3-4H parenterally.

\section{The Skeletal Muscle Relaxants}

The term is commonly used to refer to heterogeneous group of pharmacologically unrelated medications approved to treat two distinct conditions: spasticity from upper motor neuron syndromes and pain or spasms from musculoskeletal conditions such as non-specific low back pain. These include carisoprodol,chlorzoxazone, cyclobenzaprine, metaxalone, methocarbamol and orphenadrine.

- Cyclobenzaprine is the best studied drug for relieve of skeletal muscle spasms and has also shown effectiveness in the treatment of fibromyalgia. It is a centrally acting skeletal muscle relaxant with antidepressant activity. Cyclobenzaprine seems to act primarily at the brain stem to reduce tonic muscle motor activity, influencing both gamma and alpha motor neurons leading to a reduction in muscle spasms. Dosage is 15 - $30 \mathrm{mg}$ once daily.

- Methocarbamol is a central acting muscle relaxant which may inhibit carbonic anhydrase and the NMDA receptor. Peripherally it prolongs the muscle refractory period. The initial dosage is $1,5 \mathrm{G}$ four times a day.

- Orphenadrine is a nonselective $\mathrm{mACh}$ receptor antagonist and a $\mathrm{H} 1$ receptor antagonist which is used to treat muscle 
pain and with motor control in Parkinson's disease. It also is an NMDA receptor antagonist activity which may contribute to it's muscle relaxing activity. The dosage is $100 \mathrm{mg}$ two - three times daily.

A Cochrane review for acute low back pain which included 8 trials found that these drugs were superior to placebo for short term pain relief and global efficacy. It also found these drugs are associated with more total adverse events and sedation than placebo, though most events were self-limited and serious complications appeared rare.

\section{Other CNS Drugs used in Lower Back Pain}

- Antidepressants

The tricyclic antidepressants like amitriptyline are often used in the treatment of chronic back pain. They block the uptake of amines by nerve terminals. Duloxetine has been studied extensively in the use in pain conditions and is approved for the treatment of fibromyalgia and chronic musculoskeletal pain, including discomfort from osteoarthritis and chronic lower back pain.

- The benzodiazepines, specifically diazepam, is used for muscle spasms, but it is not approved for this indication.

- Gabapentin is an anti-epileptic drug which modulates the action of 2 enzymes involved in GABA biosynthesis and binds to the alpha2delta subunit of voltage gated calcium ion channels. It is recommended as a first line medication for the treatment of neuropathic pain in diabetes, Herpes and central neuropathic pain

- Pregabalin is also an anti-epileptic that acts on the calcium ion channels which is used in neuropathic pain.

The practitioner sees many prescriptions from specialists given to patients suffering from lower back pain. If the patient's pain is not well controlled, make sure that when you prescribe, at least an analgesic, a NSAID and a CNS drug used for neuropathic pain is recommended. It often helps to replace one of these drugs with a drug in the same category. There are various options strive to help the patient to get to the best control of pain.

This article was compiled from a lecture given by the author and the references are available on request. 